\title{
EchoGéo
}

11 | 2010

Madagascar

\section{Processus d'intégration de villages périphériques dans le système d'approvisionnement de Bamako}

Gwenaëlle Raton

\section{OpenEdition}

Journals

Édition électronique

URL : https://journals.openedition.org/echogeo/11559

DOI : 10.4000/echogeo.11559

ISSN : 1963-1197

Éditeur

Pôle de recherche pour l'organisation et la diffusion de l'information géographique (CNRS UMR 8586)

Référence électronique

Gwenaëlle Raton, «Processus d'intégration de villages périphériques dans le système d'approvisionnement de Bamako », EchoGéo [En ligne], 11 | 2010, mis en ligne le 24 février 2010,

consulté le 31 juillet 2021. URL : http://journals.openedition.org/echogeo/11559; DOI : https://doi.org/ 10.4000/echogeo.11559

Ce document a été généré automatiquement le 31 juillet 2021.

EchoGéo est mis à disposition selon les termes de la licence Creative Commons Attribution - Pas d'Utilisation Commerciale - Pas de Modification 4.0 International (CC BY-NC-ND) 


\title{
Processus d'intégration de villages périphériques dans le système d'approvisionnement de Bamako
}

\author{
Gwenaëlle Raton
}

\section{Introduction}

1 Bamako est une capitale sahélienne enclavée. Comme d'autres villes de la ceinture sahélienne, Bamako assure une fonction de relais entre les flux d'importations provenant des ports côtiers et l'approvisionnement de son arrière pays en produits vivriers et manufacturés. La politique de désenclavement a permis un renforcement des liaisons vers la Côte d'Ivoire, la Guinée, la Mauritanie et l'Algérie, bien que le réseau routier soit vétuste et mal adapté aux exigences de transport actuel ${ }^{1}$. L'arrière pays joue également un rôle d'approvisionnement de Bamako. Le développement de lignes de transport régulières en périphérie vers des aires de production villageoises a marqué la conversion de certains marchés villageois préexistants en pôles de rencontre entre acteurs de l'approvisionnement de Bamako.

Le rôle de la périphérie de Bamako tend à se complexifier. Fonction agricole à destination de la ville, rôle de commercialisation et de distribution, fonctions résidentielles se conjuguent désormais sur un même espace. Ce phénomène est loin d'être unique et on le retrouve dans de nombreux pays ouest africain. L'originalité de la capitale malienne tient au développement de la fonction commerciale en périphérie. On assiste en effet depuis les années 1980 à la multiplication des aires de vente rurales. Ce dynamisme commercial est le produit d'échanges entre un marché urbain pourvoyeur de produits manufacturés et une offre rurale productrice de denrées agricoles et de bois-énergie ${ }^{2}$. La rencontre de l'offre et de la demande s'effectue sur une centaine de marchés hebdomadaires appelés «foires » qui centralisent les produits et les acteurs avant une redistribution multidirectionnelle allant du marché de gros à Bamako, aux petites boutiques villageoises ou aux vendeurs ambulants. Les foires font 
figures à la fois de point de rupture de charge périodique et de premier échelon d'accès aux biens non produits in situ pour les populations rurales. Elles constituent donc des places centrales, bien que peu complexes en termes de marchandises et de services délivrés, puisqu'elles visent à écouler les productions locales (surplus) vers la ville et à la satisfaction des besoins courants des ruraux.

3 Les foires sont les lieux visibles de la mise en relation des commerçants et des producteurs. «C'est là que peuvent être repérés le plus facilement les acteurs et les flux de marchandises, étudiés les prix, jalonnés les circuits, localisées les aires de production" (Chaléard, 2002, p.11). D'autres flux, plus discrets complètent l'approvisionnement urbain. Il s'agit d'un approvisionnement direct sur les sites d'exploitation (agricoles ou forestiers) sans passage sur une aire commerciale. Il est souvent la conséquence de partenariats anciens entre commerçants et producteurs, issus de rencontres sur une foire.

4 On recense les premières foires en périphérie de Bamako en 1960, lorsque le Mali accède à l'Indépendance. Cependant, quelques unes d'entre elles sont précédées d'une expérience commerciale, en tant que marché villageois à faible rayonnement ${ }^{3}$. Depuis, la foire s'est imposée comme un mode de commerce innovant, en phase de normalisation dans toute la périphérie. Pour les commerçants urbains, elle constitue un lieu incontournable de concentration de marchandises issues de l'agriculture locale (inédit à l'échelle de la périphérie). Pour les ruraux, la foire est un débouché de proximité qui permet la vente des surplus de la production familiale. Seule la constitution de solides réseaux d'information et de fidélisation permet aux commerçants ou producteurs de la zone de les contourner. Les acteurs les plus expérimentés tentent de cette façon d'éviter la concurrence, inconvénient majeur de l'aire commerciale, largement compensé selon bons nombres d'acteurs par la quantité de marchandises disponibles, résultat de la concentration hebdomadaire de producteurs.

5 La polarisation de flux induite par la présence d'une foire est devenue un argument de poids en termes de gouvernance incitant à la multiplication de ce mode de commerce. En effet, depuis la mise en place de la décentralisation au Mali, on assiste à une appropriation communale de ces marchés hebdomadaires dont les retombées économiques sont de mieux en mieux perçues. La mise en place de la décentralisation a marqué un tournant et a légitimé ce type d'aire de vente comme structure attractive, offrant une source de revenus à l'échelle communale. Chaque commune étant désormais désireuse d'avoir sa propre foire, la forme du réseau de foires est donc loin d'être achevée.

6 Cependant la multiplication des foires n'est pas un phénomène inéluctable. Le jeu de la concurrence entre les foires agit comme un régulateur économique. En effet, que leur création soit le fait d'un long processus de développement issu d'échanges entre groupes sociaux nouant des liens anciens ou qu'elle soit l'objet d'une politique communale, la durabilité d'une foire n'est garantie que lorsqu'elle est source d'émission de flux de marchandises. Ainsi, en périphérie de Bamako, le réseau de foires est évolutif. Il est soumis à de nouvelles dynamiques, tantôt créatrices ou destructrices, d'un ou plusieurs maillons du réseau.

7 Le phénomène le plus intéressant dans la diffusion de ce mode de commerce est sans doute que, par sa seule fonction d'échange, la foire joue un rôle dans l'intégration d'unités agricoles et de villages au circuit urbain. Elle constitue donc un espace 
intermédiaire d'intégration au marché urbain. Maillon du circuit urbain en périphérie, la foire est une vitrine des productions locales, ainsi qu'un vecteur d'opportunités commerciales pour des communautés rurales non encore intégrées au marché urbain. Ainsi chaque semaine, une foire attire en moyenne les producteurs de quinze villages différents.

Les comportements des paysans en termes d'échanges ont évolué et l'attrait du marché urbain s'est considérablement renforcé, multipliant ainsi le nombre d'acteurs impliqués dans l'approvisionnement urbain, les quantités disponibles et la régularité de l'approvisionnement. En périphérie de Bamako, la foire est aujourd'hui la forme la plus évidente d'intégration de nouveaux villages périphériques au circuit urbain. Le constat de l'inégale répartition des foires en périphérie de Bamako, nous amène à nous interroger sur ce processus.

A travers l'étude de politiques publiques agricoles ou urbaines ciblées, nous tenterons d'identifier les mécanismes d'intégration d'exploitations familiales à l'approvisionnement urbain. Dans cet objectif, nous proposons dans un premier temps une lecture de la répartition des foires à la lumière des actions politiques antérieures, sur un temps long. Nous verrons ensuite que ces politiques posent les conditions de densification et d'extension d'un modèle marchand, celui des foires. Enfin, nous analyserons le lien de réciprocité entre développement urbain et développement commercial en périphérie.

\section{Des stratégies coloniales de conquête des espaces périphériques qui marquent durablement les terroirs}

10 Un recul historique est nécessaire pour percevoir les mutations des fonctions de la périphérie. Leur prise en compte est essentielle puisqu'elles ont modifié les comportements des producteurs en termes d'échanges, notamment dans leurs stratégies de ravitaillement de la ville. Nous nous intéressons particulièrement aux sites agricoles qui sont nés des politiques coloniales, dont le rôle s'est renforcé à l'Indépendance et qui constituent aujourd'hui des lieux actifs de fourniture de produits pour la ville. Ils sont des lieux d'occupation anciens, très tôt orientés par des politiques agricoles ou urbaines cherchant à créer des espaces fonctionnels. L'évolution de leurs fonctions nous permettra de discuter des phases d'intégration de villages aux circuits urbains. A la lumière des écrits sur ces périodes, nous apposerons un nouveau regard sur la périphérie, celui de la connaissance des aires commerciales villageoises, de leur localisation, de leur fonctionnement depuis 1960, issues des enquêtes de terrain.

\section{Rôle de l'activité maraîchère dans l'évolution des rapports de la ville avec son arrière pays}

11 La périphérie de Bamako participe à l'approvisionnement de la ville depuis la colonisation. Les rives du Niger et les abords de la ville ont très tôt été sollicités pour la production de maraîchage. Les colonisateurs ont rapidement pris conscience des potentialités agricoles du site et les ont valorisées.

Dès la période coloniale et surtout à partir de 1920, les mesures liées à la sécurisation du ravitaillement de la ville de Bamako, alors capitale du Soudan français, se 
multiplient. Les différentes fonctions administratives, militaires, économiques et commerciales assuraient alors une croissance démographique continue de la ville. Ce phénomène a pris une ampleur conséquente à partir de 1945 (Fig.1). À cette époque, Rossi Villien recense 37000 habitants, dont environ $4 \%$ de population étrangère ${ }^{4}$, liée à la présence coloniale. La demande européenne est alors exigeante ${ }^{5}$ et les efforts déployés pour la satisfaire importants. L'administration coloniale fait le choix de développer ces produits sur place. Les difficultés liées à l'importation de produits européens ${ }^{6}$ sont en effet nombreuses, et justifient la mise en place d'infrastructures pour répondre à ces besoins. Les premières actions de valorisation de la périphérie découleront de cette décision Durant cette période, la vulgarisation des produits maraîchers européens est rapide et efficace: jardins pédagogiques, fermes écoles, centres de recherches agronomiques, initiation à la consommation encouragent de nombreux acteurs à s'investir dans le secteur. C'est le cas des membres de l'administration coloniale, des fonctionnaires et militaires, ainsi que des populations autochtones qu'ils emploient et qui diffusent les techniques au gré de leurs déplacements (forcés ou non).

Figure 1 - Evolution de la population de Bamako de 1884 à 1998

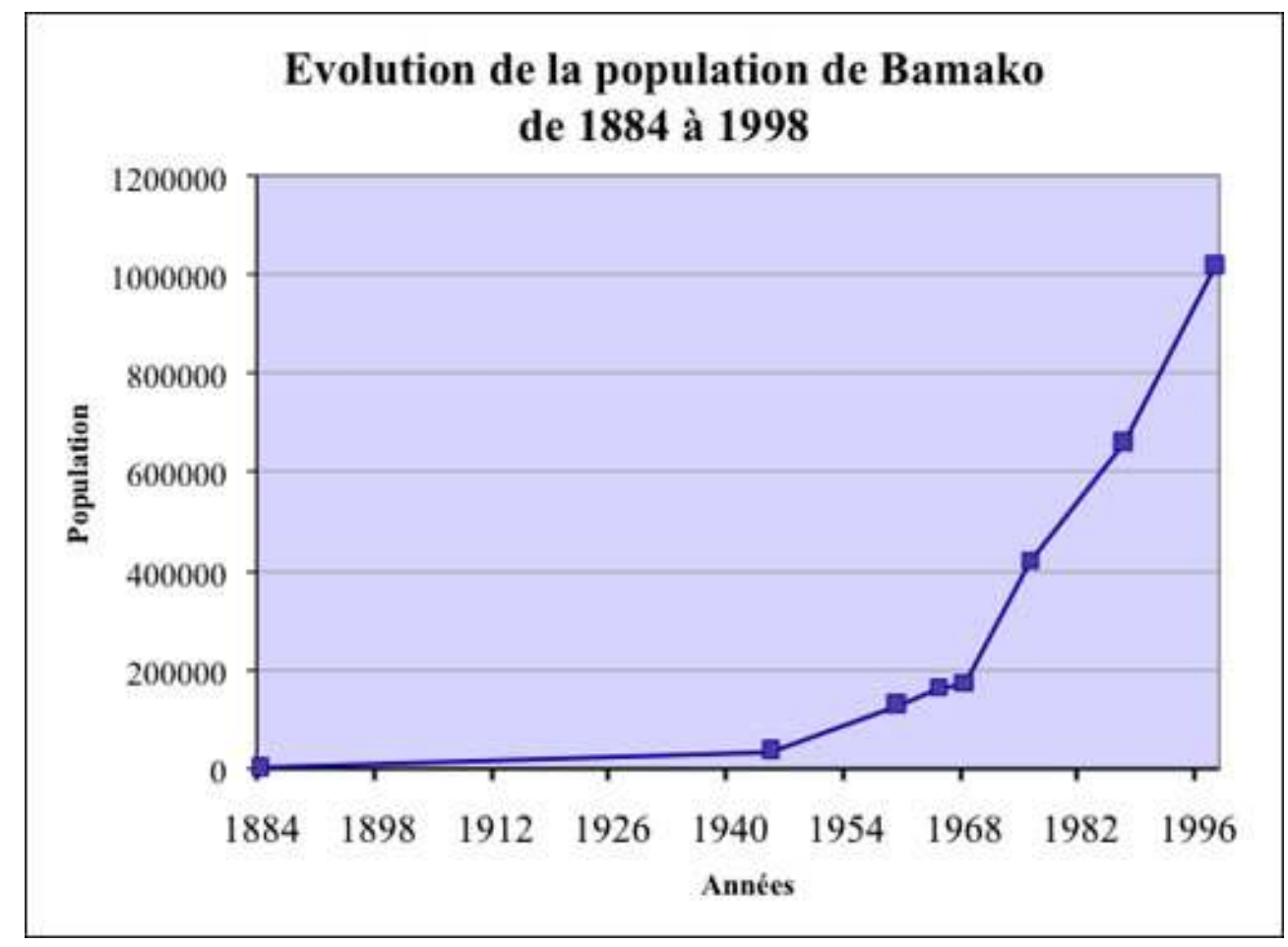

Source : Rossi Villien 1960, enquête de l'INSEE eéalisée à partir de sondages, recensement du service de la statistique du Mali, Bleneau et La Cognata

La pratique de cultures dites maraîchères précède la période coloniale. Cependant, avant l'arrivée des colons, les cultures de fruits, feuilles ou légumes s'apparentaient plus à des plantations communautaires où les arbres et plantes traditionnels étaient valorisés pour l'autoconsommation qu'à une véritable culture de potager (Doumbia, 1982, p. 57). Le passage de la culture de plantes, rentrant dans la gamme des condiments consommés, à la culture commerciale a eu lieu pendant la période coloniale. Les premières expérimentations sur la production de produits maraîchers ont été concluantes et ont permis d'assurer l'approvisionnement de l'administration 
coloniale implantée sur le site de Bamako. Ces premiers succès ont donné lieu à un investissement plus important selon deux idées fixes : la première était de favoriser l'implantation de l'activité agricole par fixation de la population (forte densité) et la seconde de favoriser l'intégration d'une paysannerie innovante aux circuits de ravitaillement urbain. La valorisation agricole de la périphérie, basée sur ces doctrines, explique sans doute la fixation rapide d'activités agricoles. Durant la première phase d'expérimentations agricoles datant des années 1920, les infrastructures liées aux activités de maraîchage sont présentes en centre ville et dans les interstices urbains, ainsi que sur trois des six axes routiers menant à Bamako dans un périmètre n'excédant pas $15 \mathrm{~km}$. La création d'un périmètre irrigué à $30 \mathrm{~km}$ à l'est de la capitale, sur la rive droite du Fleuve Niger, à proximité de la route menant vers Ségou, marque l'implantation coloniale sur un quatrième axe, dans un périmètre plus large.

14 Après une période de diffusion du maraîchage, ces travaux montrent le passage à un autre objectif. Il s'agissait pour les administrateurs coloniaux de valoriser un espace à fort potentiel productif, d'une part pour y mener des activités de maraîchage mais plus largement pour y développer l'agriculture de rente. Bien que les objectifs soient différents, les stratégies utilisées pour permettre le développement du périmètre irrigué de Baguinéda ${ }^{7}$ sont similaires à celles vues précédemment. Pour l'administration coloniale, ces aménagements devaient induire des changements importants au sein des espaces ruraux. In Colonisation Indigène, "The French combined their belief in the importance of high population density with their convictions regarding the superiority of intensive plow agriculture and the centrality of private property and the nuclear family to a developed agricultural system" (Van Beusekom, 1997, p. 303). L'aménagement du périmètre irrigué de Baguinéda répond à ces critères. Une dizaine d'années après les premiers travaux (1936), on recensait sept villages. Encadrés par des techniciens et des administrateurs urbains, ces espaces sont devenus des lieux d'innovation et de transmission des savoirs et techniques agricoles. L'augmentation progressive du nombre de villages montre que les techniques des administrateurs ont été payantes en matière de fixation de la population et que l'activité agricole s'est diffusée. Dès lors, le transport forcé n'est plus une condition nécessaire au développement des activités agricoles.

Ces observations mènent au constat suivant: si la culture d'exportation a été un outil stratégique de valorisation économique de l'espace pendant la colonisation, à Bamako plus qu'ailleurs le maraîchage est devenu un fondement de la vocation de la périphérie proche. En effet, en tant que foyer important de Français, les espaces périphériques proches ont eu pour fonction prioritaire le ravitaillement de la ville. Bien que la présence du périmètre irrigué de Baguinéda montre la volonté d'une ouverture vers les productions de rente, il fut important pour les administrateurs coloniaux de garantir la sécurité alimentaire des acteurs administratifs et politiques attestant de la présence coloniale. D'autre part, la position stratégique de Bamako et son affirmation comme capitale coloniale confirma son rôle de contrôle des flux d'exportation plutôt qu'un rôle de production intensive in situ. A l'époque coloniale, deux fonctions principales de la périphérie proche de Bamako peuvent être dégagées : d'une part la priorité est donnée aux fonctions de ravitaillement de la ville, en particulier en produits maraîchers, d'autre part les activités de la périphérie font office de vitrine de la production soudanaise. Bamako contrôle non seulement les flux de l'agriculture de rente, mais diffuse l'innovation auprès des communautés paysannes. 
16 La demande citadine, celle des colonisateurs en particulier, a donc stimulé les productions agricoles de la périphérie et a entraîné la mutation d'une agriculture d'autoconsommation en une agriculture insérée dans le marché intérieur, en particulier celui de la ville. Le développement du maraîchage en périphérie de Bamako a impulsé l'intérêt pour le marché urbain, bien qu'il ne concerne à cette époque qu'une partie des communautés paysannes périphériques. La présence des Français a eu pour conséquence de stimuler, en périphérie de Bamako, une agriculture ayant pour débouché la ville, plus qu'une intégration aux marchés d'exportation. Cette époque marque une première rupture des comportements des paysans en termes d'échanges.

\section{Les politiques de mise en valeur de la périphérie sont propices à la diffusion des foires}

17 Comme nous l'avons vu précédemment, les foires apparaissent sous leurs formes actuelles à partir de l'Indépendance. Pourtant, une analyse rétrospective de la période coloniale montre que les bases de la diffusion d'un modèle commercial sont présentes.

Durant l'époque coloniale, la vulgarisation des cultures maraîchères ne s'est en effet pas limitée à la formation et aux travaux agricoles. L'administration coloniale a introduit chez les populations autochtones les «foires-expositions». Etablies dans les capitales régionales ${ }^{8}$, elles sont présentées comme de véritables «vitrines» de l'horticulture où les meilleurs agriculteurs et les techniques les plus performantes étaient récompensés. Prenant appui sur les marchés villageois déjà existants, ces foiresexpositions ont renforcé le rôle de la capitale soudanaise et développé «l'esprit de prestige » lié au maraîchage. C'est l'ensemble de la région bamakoise qui a bénéficié de cet engouement pour l'activité. Dans la lignée des politiques de fixation d'une population agricole et de leur formation, les foires ont constitué un outil pour insérer durablement l'innovation agricole dans les pratiques.

De plus, la politique de vulgarisation des activités maraîchères a ciblé une gamme d'acteurs différenciés, enclins à perpétuer les techniques pour leur propre compte et à les diffuser. Dans chacun des sites développés, le prestige lié aux cultures maraîchères s'est maintenu. Les anciens militaires furent garants des pratiques de cette activité et ont fortement participé à la diffusion des techniques. D'ailleurs, chaque base militaire française a donné naissance à des sites spécialisés dans un ou deux produits maraîchers. C'est le cas de Kati, près du camp militaire, qui n'a cessé depuis l'arrivée des colons d'affirmer son rôle de pôle maraîcher, avec des spécialisations telles que la pomme de terre et la patate douce. La foire-exposition, qui fût l'une des plus importantes avec Bamako, n'existe plus aujourd'hui sous cette forme, mais a muté en deux marchés hebdomadaires appelés foires : l'un se tient le jeudi, l'autre le dimanche. On retrouve aussi des sites spécialisés dans les produits maraîchers à Koulikoro, à proximité de l'ancienne base militaire française. La présence de l'Institut Polytechnique Rural (IPR) de Katibougou ${ }^{9}$ et d'une exploitation des rives du Niger a renforcé la spécialisation.

Les foires-expositions ont ancré ces pratiques. D'ailleurs, il est intéressant de se pencher sur cette terminologie. On peut se demander si le terme français « foire » n'a pas été introduit à cette époque. Son utilisation très courante actuellement, pour désigner les marchés hebdomadaires villageois, ne renvoie-t-il pas à cette notion d'exposition des capacités agricoles, du rôle innovant et du prestige lié au maraîchage? 
Bien que l'activité maraîchère ne soit pas la spécialité unique de ces marchés ${ }^{10}$, il semble que le bagage idéologique et théorique lié à l'emploi de ce terme ait subsisté. Cela pourrait expliquer pourquoi l'emploi du mot «foire » se soit substitué au terme «marché » dont la périodicité semble plus proche de la définition usuelle. La diffusion de ce mode de commerce: la foire, puis sa normalisation depuis les années 80 trouverait ainsi son origine dans les premières politiques de valorisation de la périphérie.

\section{L'histoire urbaine explique le sens de propagation de ce modèle commercial}

21 La localisation des sites maraîchers pendant la période coloniale est primordiale pour comprendre la structuration des espaces agricoles fonctionnels aujourd'hui. Jusqu'à 1960, la diffusion des activités de maraîchage s'étend en périphérie proche de Bamako, sur un modèle auréolaire assez classique.

22 A l'accès à l'Indépendance, la périphérie de Bamako dispose de nombreuses structures agricoles : écoles, ferme d'Etat, espaces de plantation, centre de recherche fruitière. La politique socialiste de Modibo Keita, premier président du nouvel Etat Malien, a consisté à utiliser ces lieux pour faire croître la vocation agricole, dans l'objectif de diminuer les effets d'un exode rural jugé alors comme excessif et dangereux. Les premières années de l'Indépendance s'inscrivent en effet dans une période où les débats scientifiques et non scientifiques s'ouvrent sur l'influence néfaste d'une urbanisation incontrôlée. «En état de sururbanisation, les pays du sud étaient engagés sur une trajectoire d'échec: celle-ci conduisait d'une part au triomphe de mégapoles macrocéphaliques ingérables et qui anémiaient les échelons inférieurs des armatures urbaines, d'autre part à la ruine des campagnes et des agriculteurs contraints de quitter la terre pour tenter de survivre dans des villes sans bases économiques viables " (Chaléard et Dubresson, 1999, p. 8). Le phénomène d'exode rural constaté dans les pays du sud pousse à un constat alarmant. Une des priorités du jeune Etat est de juguler la croissance des villes en émettant l'hypothèse qu'elle ne constitue pas seulement un risque de surconcentration urbaine, mais également de perte des valeurs rurales et du travail agricole. On sait désormais que ce phénomène d'exode rural n'a pas participé au dépeuplement des espaces ruraux : «La population rurale en Afrique a en gros triplé en un demi-siècle, passant de 149 millions de personnes en 1950 à 442 millions en 2005 (d'après la FAO) » (Chaléard, 2006). Cependant la période d'accès à l'Indépendance reste marquée par un brusque accroissement annuel qui a sans doute joué un rôle important dans les prises de décisions politiques. "Si la croissance démographique de Bamako s'est poursuivie jusqu'à nos jours, jamais elle n'aura présenté un taux d'accroissement annuel aussi élevé qu'au cours de cette période » (Piche, Ouedraogo, 2000, p. 16). 
Figure 2 - Circulaire du ministère de l'Intérieur relative au « retour à la terre » au Mali en 1962

Figure 2

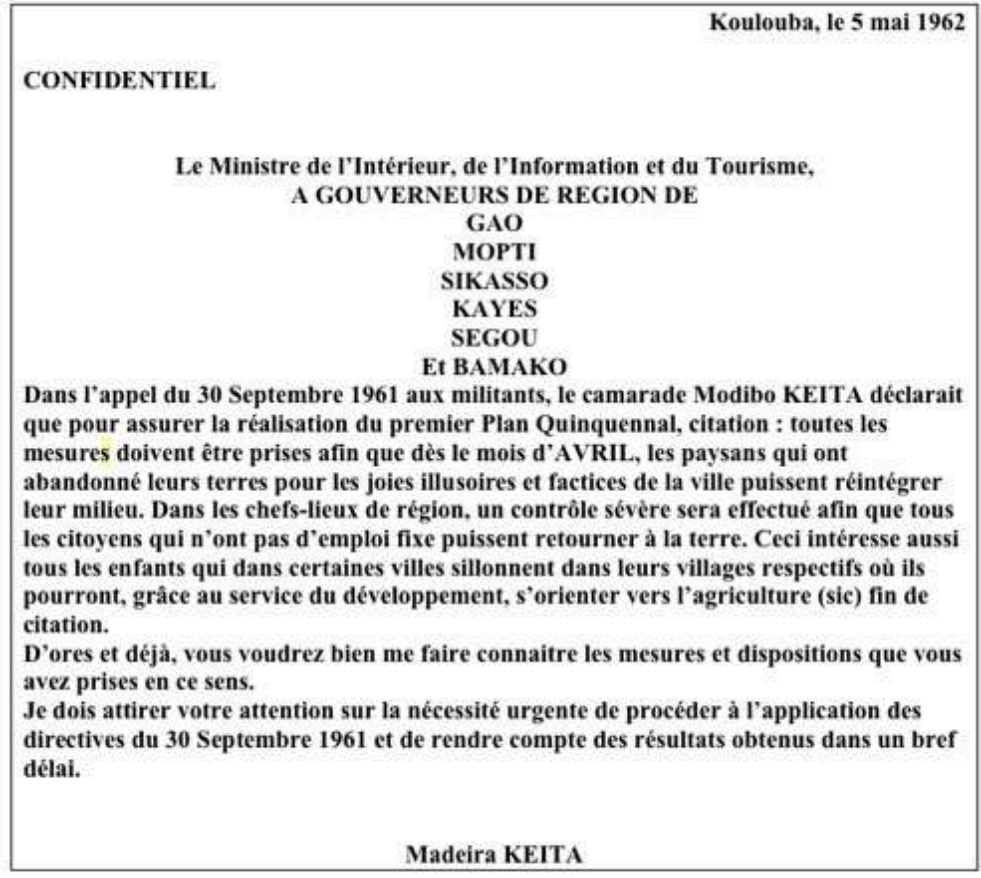

Source: (GARY-TOUNKARA, 2008, p205)

Source : Gary-Tounkara, 2008, p. 205

Dans ce contexte, la politique socialiste de Modibo Keita met en place la lutte contre l'exode rural. Des villages appelés «villages de colonisation» ou «villages de concessions rurales » sont créés en périphérie proche ${ }^{11}$. Ils sont fondés sur le principe des villages de colonisation du périmètre irrigué de Baguinéda. Peu utilisés avant l'Indépendance, ils s'apparentent dès lors à un outil de fixation agricole pour des acteurs privés. Il n'y a aucun recours à une main d'œuvre forcée, les droits d'usage et d'occupation sont concédés par une procédure d'attribution foncière. La spécificité de ces attributions foncières est liée au statut des terrains ruraux, qui sont attribués sous forme de concessions rurales sans immatriculation, alors qu'elle est obligatoire pour l'affectation des autres terres de l'Etat.

24 La concession rurale est un enclos familial qui comprend un ou plusieurs bâtiments agricoles. Les limites du parcellaire sont matérialisées par des murets en durs, des plantations (eucalyptus) ou encore des haies mortes. La figure 3 illustre la matérialisation des limites du parcellaire, qui est une des préoccupations majeures des propriétaires une fois les démarches administratives engagées. Les concessions rurales se distinguent aisément dans le paysage de l'habitat traditionnel regroupé en village tas, ou des hameaux permanents ou de culture. L'intérieur de la concession est aménagé avec soin, séparant les bâtiments agricoles du petit élevage de bovins ou de volailles, les cultures fruitières ou maraîchères des cultures d'hivernage. Le travail agricole effectué dans les fermes citadines est assuré par de la main d'œuvre salariée au service d'urbains, fonctionnaires ou commerçants. Occupés par leurs activités professionnelles, ils ne sollicitent pas en général la sphère familiale, sauf pour des déplacements ponctuels, le week-end surtout. A cet effet, on remarque souvent un petit 
logement d'appoint permettant d'accueillir le propriétaire ou ses salariés. La majorité des concessions rurales est exploitée en cultures maraîchères ou arboricoles.

Figure 3 - Construction d'un muret en parpaing à Saala, dans la commune de N'Gabakoro Droit

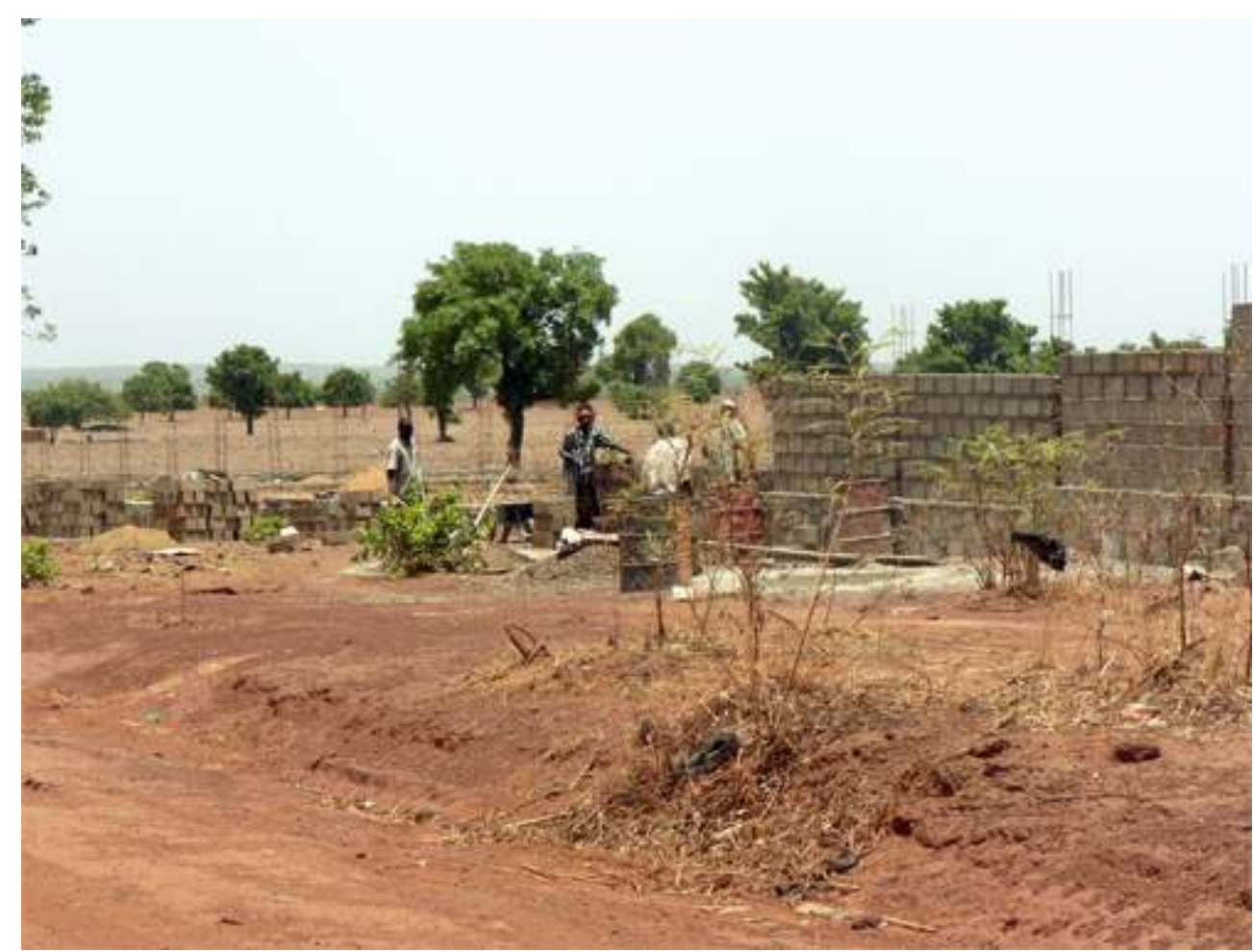

La matérialisation des limites du parcellaire est une des priorités des nouveaux propriétaires de concessions rurales. On observe dans cette concession un parc de mangiers er de karité, ainsi que les résidus de cultures de mil

Source : G. Raton, juin 2007

Ces actions, initiées par souci d'équilibre régional, n'auront pas les résultats escomptés. En effet, afin d'inverser les flux migratoires, ce sont les citadins qui sont encouragés à mettre en valeur des parcelles, au détriment des communautés paysannes. Ces citadins, sans emploi, sont encouragés dans leurs démarches, formés aux techniques culturales, fournis en matériel agricole. Ce système est en fait une copie des écoles rurales de l'époque coloniale, si ce n'est que l'Etat leur fournit la terre de manière durable. Malgré des incitations sociales, la lourdeur des démarches administratives en fait rapidement un outil d'acquisition de terre pour une minorité urbaine aisée. La main mise des notables, usant de leurs relations, est dénoncée mais se renforce. La majorité des propriétaires de concessions rurales à cette époque sont commerçants, membres du gouvernement ou militaires (Doumbia, 1982, p. 107).

L'émergence de concessions rurales révèle quatre processus. Le premier est l'importance de la localisation près des axes routiers actifs. En 1960, les axes fréquentés par les camions et camionnettes (type véhicules bâchés) sont ceux valorisés par l'administration coloniale: la route à l'est de Bamako menant vers Ségou (via Baguinéda), la route menant à Koulikoro, l'axe Kati, et la route menant à la frontière guinéenne (via Samanko). Les villages de concessions rurales s'implantent massivement sur ces axes, afin de faciliter la communication et la diffusion des produits vers le marché urbain. 
déjà tournés vers le maraîchage et soumis à la pression urbaine à court terme. En effet, d'autres zones font l'objet d'attribution dans la continuité des infrastructures implantées pendant la colonisation: à Moribabougou, en amont de l'école de Katibougou et le long du fleuve du Niger, zones déjà largement valorisées à cette époque pour le maraîchage; à Samanko où une plantation d'Etat fut implantée et continue d'être développée pour des intérêts privés.

Le troisième processus est l'émergence de marchés appelés " foires » dans les villages de concessions rurales. Ceci révèle une appropriation par les communautés paysannes des foires-expositions de l'époque coloniale, avec cette fois-ci une implantation spatiale qui passe des capitales régionales aux terroirs villageois. On peut citer l'exemple de Kassela, qui est un village d'exploitation situé sur la route principale menant à Ségou, après Baguinéda, dont il est distant d'une quinzaine de kilomètres. Dès les années 1960, il hébergeait une foire et profitait ainsi de la proximité d'un axe routier fréquenté pour écouler régulièrement la production vers Bamako. Kassela se situe à proximité de la forêt classée de La Faya, ce qui encourage, dès la création du marché, la commercialisation du bois énergie vers la ville.

Enfin, le dernier processus est celui du mitage de l'espace rural créé par le lotissement d'espaces agricoles de type « concession rurale ", respectivement à 40 et $60 \mathrm{~km}$, au sein de pistes non goudronnées, que nous appellerons "pistes rurales». Ces pistes desservent les villages de la périphérie les plus reculés. Ce mitage de l'espace contraste fortement avec le schéma auréolaire d'extension des parcelles agricoles observé pendant la période coloniale et marque les débuts d'une extension ponctuelle des infrastructures agricoles et des aires de vente, sans nécessaire continuité avec les mises en valeurs précédentes. C'est le début d'une différenciation spatiale en périphérie entre espaces inclus dans le circuit urbain et espaces en dehors de ces réseaux marchands.

Notons que le principe d'attribution de concessions rurales par l'Etat reprend les deux axes de valorisation de l'espace utilisés pendant la période coloniale. D'une part, créer des espaces agricoles fonctionnels en valorisant la recherche et la transmission des techniques, d'autre part en faire des pôles d'innovation et de diffusion de l'activité. L'objectif affiché pendant la période coloniale, qui consistait à faire de la périphérie de Bamako une vitrine des potentialités agricoles, est remplacé par le but principal du jeune gouvernement : valoriser le travail de la terre et inciter au retour à la vie rurale.

31 L'introduction d'une élite urbaine dans le milieu agricole par le biais des concessions rurales a initié une proximité entre deux communautés qui avaient peu de contacts. Dans un contexte de valorisation culturelle de l'urbanité (on sait que l'exode rural, s'il n'a pas marqué durablement le paysage rural, correspond à une vague d'intérêt pour le milieu urbain), la proximité d'exploitations agricoles «modernes» a sans doute encouragé l'étalage des capacités agricoles de la part des communautés paysannes. On comprend alors pourquoi la création de foires hebdomadaires a été concomitante de l'installation des villages de concessions rurales, sans qu'on puisse attribuer cette création à une des deux « communautés ». La politique de retour à la terre a donné aux notables urbains l'occasion d'investir dans l'agriculture, dévalorisée socialement, mais avec les outils qui étaient alors les leurs : appropriation foncière, usage d'ouvriers agricoles et délimitation des lots de culture. Paradoxalement, cette main mise urbaine a joué un rôle important dans la diffusion des techniques agricoles et dans la perception de la ville comme débouché économique pour des sociétés paysannes jusque là centrées 
sur l'autoconsommation. Les communautés paysannes proches étaient sollicitées pour le travail salarié, elles apprenaient ainsi les techniques de production, la gamme des produits commercialisables et les prix. Insérés dans des terroirs villageois, ces fermes citadines ont suscité l'intérêt, d'autant plus que les propriétaires représentaient l'élite urbaine, moderne et aisée.

\section{Le secteur artisanal normalise la foire comme débouché des productions locales et organise la périphérie}

\section{Evolution des comportements des paysans en termes d'échange et intégration massive au marché urbain}

En périphérie de Bamako, le cap de l'économie de subsistance est aujourd'hui dépassé. La proximité de l'agglomération en tant que principal agent de diffusion monétaire, a joué un rôle dans la transformation des échanges. Cependant, il faut noter que coexistent différentes échelles d'échanges qui n'ont pas toujours intégré le marché urbain. A grande échelle, il s'agit des échanges tels qu'ils sont induits par l'économie de subsistance, c'est-à-dire une fonction d'échange local pour satisfaire des besoins non produits par un groupe et échangeables par un autre. A l'échelle nationale, il s'agit des échanges liés à la complémentarité entre des espaces producteurs de surplus et des espaces déficitaires. Ils s'incèrent dans les flux internes du pays et peuvent revêtir des spécificités liées aux types de produits. Dans ce cas, les conditions physiques du milieu peuvent expliquer des spécialisations.

L'intégration d'unités agricoles et de villages dans les circuits urbains est le fruit de l'évolution des comportements des paysans en termes d'échange. En périphérie de Bamako, ces comportements ont été influencés par des politiques agricoles ou foncières, qui ont favorisé la commercialisation des productions vers la ville. Désormais, les échanges sont marchands, ils sont pratiqués au sein de marchés et ils complètent la production familiale dont une portion reste cependant vouée à la sécurisation des besoins alimentaires. Les pratiques de commercialisation d'une partie des productions familiales vers la ville ont consolidé l'intégration dans les circuits urbains des terroirs proches.

C'est en ce sens que les villages de concessions rurales créés à l'Indépendance ont eu un rôle important. Bien que les communautés paysannes aient été à priori désavantagées par la main mise urbaine sur le capital agraire de la périphérie, on peut constater que cet outil a permis le développement de circuits parallèles de ravitaillement de la ville, nés de l'initiative paysanne. Le maraîchage s'est développé au sein des unités de production familiales, sur de petites superficies appelées jardins. Il s'agit de champs individuels cédés ou prêtés selon les usages coutumiers. Ce sont d'abord les espaces proches des concessions rurales qui ont été concernés, ainsi que les espaces situés près des voies de communication et des bonnes terres non appropriées par les citadins. La fréquentation des foires constitue pour ces producteurs la suite logique de leurs stratégies de production. Cette tendance s'accentuant, on note une modification des comportements commerciaux qui interpelle sur le discours dominant. En effet, bien que, dans les discours, les paysans valorisent l'importance de l'autoconsommation ${ }^{12}$, la 
proximité du marché urbain et la multiplication des réseaux de distribution incitent de nombreux agriculteurs à commercialiser une partie toujours plus importante de leurs marchandises et à orienter le type de production en fonction de la demande urbaine. Ce phénomène peut paraître classique. Il est ici intéressant dans le sens où il est localisé en continuité des villages de concessions rurales et qu'il permet de différencier des degrés d'intégration au marché urbain. En effet, en périphérie de Bamako, les espaces particulièrement bien fournis en aires de vente (l'axe Mountougoula/Tyélé, les lieux de production situés entre les deux axes Banamba et Kolokani, l'ensemble des villages à proximité de Siby, voir fig. 4) sont l'objet de productions intensives qui justifient la fréquentation de 2 à 4 foires par semaine. Le discours dominant peut donc être tempéré par des pratiques qui s'approchent doucement, dans certains espaces, d'une commercialisation totale de la production. Les paysans préférant de plus en plus percevoir en monnaie le fruit de leur travail et l'utiliser à leur guise.

Figure 4 - Carte des zones de productions où la commercialisation vers la ville est au moins bihebdomadaire

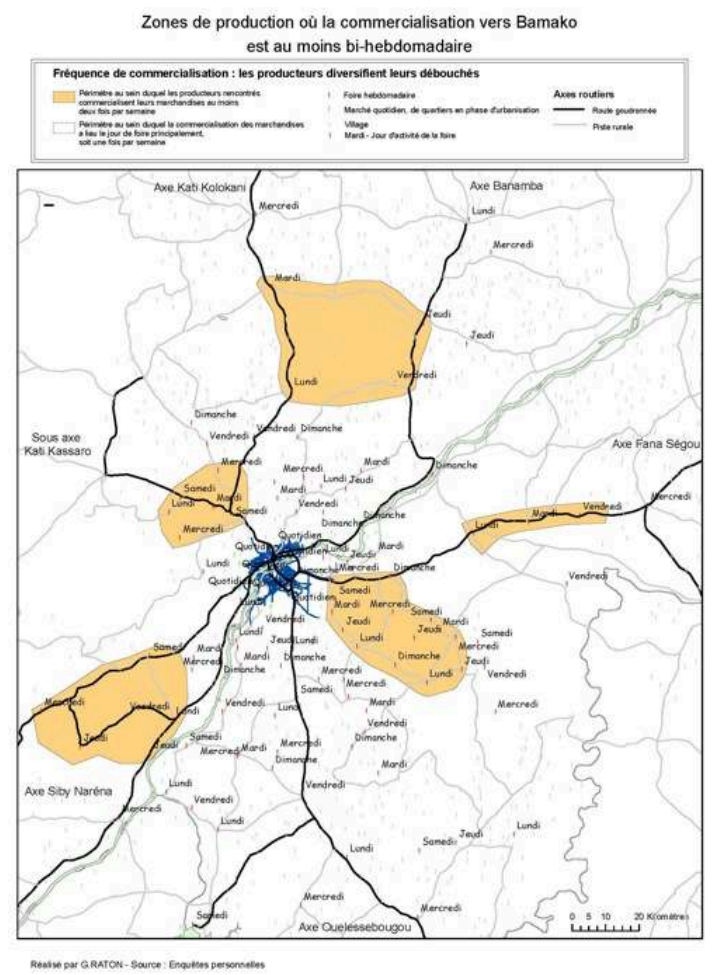

Source: G. Raton

Les zones de forte densité de foires ne sont pas encore suffisamment développées pour permettre une généralisation de ces pratiques (fig. 4). Cependant, cela confirme une tendance à la hausse. Ces pratiques montrent que la densification des flux est désormais perceptible dans les paysages de la périphérie, ainsi que leur intégration au circuit urbain. Etant donné la tendance à la concentration vers les foires, ces espaces soumis à une intensification des flux vont voir croître leur rôle de place centrale. 


\section{Ancienneté de la mise en valeur et lieux centraux} colonisation, les débuts de l'urbanisation massive, l'orientation vers les cultures d'exportation sont des étapes importantes pour comprendre la constitution de réseaux d'approvisionnement. Le choix du travail sur une longue période est justifié par un constat: sur le terrain, les villages accueillant les foires les plus attractives, les plus citées par les commerçants et les mieux desservies sont connues et fonctionnelles depuis l'Indépendance et ont pour partie une origine coloniale. Non seulement les mises en valeur premières de la périphérie sont lisibles sur le réseau de foires, mais en plus, ces sites sont devenus des lieux centraux de l'approvisionnement de Bamako. Leurs fonctions, bien qu'elles aient parfois changé, restent très fortement marquées par ce passé.

D'autres processus que ceux évoqués permettent de comprendre cette dynamique. Tout d'abord, des processus fonciers. En effet, la politique de valorisation des espaces cultivables de la périphérie proche va de pair avec la spéculation sur les terrains urbains. De nombreux citadins ont compris que le statut de concession rurale était non seulement un moyen de diversifier ses revenus en investissant dans le domaine agricole, mais également la possibilité d'accéder au titre foncier définitif. Les espaces situés dans un rayon de $10 \mathrm{~km}$ du centre ville sont urbanisés, de nombreux villages suburbains sont intégrés à la ville ${ }^{13}$ et l'on assiste de plus en plus à la transformation de concessions rurales en habitat urbain. L'opportunité d'être propriétaire de ces espaces apparaît comme lucrative et engendre une ruée sur l'immobilier.

Les infrastructures routières ont également joué un rôle important dans la localisation des zones de production et leur intégration au réseau de foire. Les circuits réguliers des «bâchés ${ }^{14}$ » instaurent des arrêts dans les marchés hebdomadaires comme Baguinéda et Kassela. A partir de 1960 et selon nos entretiens, cet approvisionnement sur les foires s'additionne à des approvisionnements réguliers vers d'autres axes. La présence de Samanko Plantation sur l'axe menant à la Guinée, puis de Ouinzindougou (village de concession rurale) a favorisé la culture de plantation sur cet axe. Les unités agricoles qui investissent dans ce secteur ont créé la même année une foire à Siby, afin de faire face à l'augmentation des volumes de marchandises. Cette foire est désormais le principal pourvoyeur de mangues de toute la région de Koulikoro avec Baguinéda. Enfin, la valorisation graduelle des axes de communication se clôt par l'exploitation nouvelle de la route menant à Sikasso. La mise en place de flux réguliers de produits issus d'une agriculture plus humide comme le manioc, la pomme de terre et l'oignon vers Bamako fait apparaître des opportunités commerciales pour les sociétés paysannes situées au sud de Bamako, près du fleuve Niger. Les circuits des «bâchés " s'ouvrent donc sur cet axe, desservant Sanankoroba, Dangassa et Sélingué. A cette époque, on note aussi l'extension des zones de production anciennes de Kati vers le nord, avec l'émergence de deux foires : Nossombougou et Yélékébougou (fig. 5). 
Figure 5 - Carte des foires en périphérie de Bamako

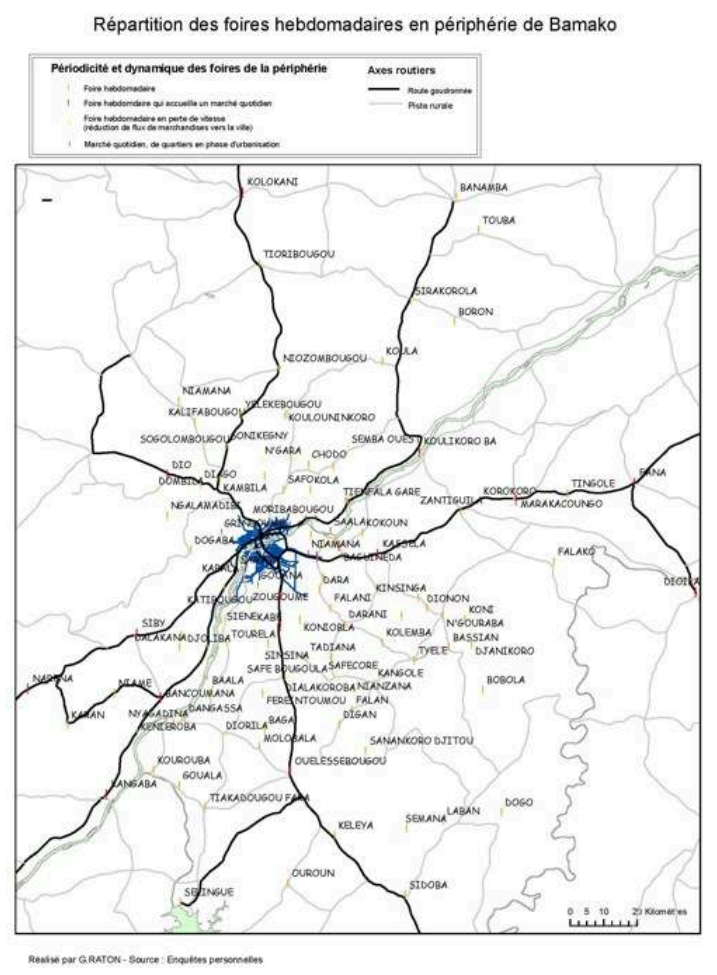

Source : G. Raton

39 La capacité d'attraction des foires s'est accrue depuis leur création (1960). Bien que, nous l'ayons vu, cet engouement pour ce mode de commercialisation soit le résultat de politiques antérieures, son extension en périphérie de Bamako, est le fruit d'initiatives paysannes, celles du secteur artisanal. En effet, la constitution d'un véritable réseau d'approvisionnement s'opère dans un contexte économique libéral où les flux de marchandises, n'étant plus organisés par l'Etat, font l'objet d'une organisation spontanée, adaptée aux pouvoirs d'achat des acteurs de l'approvisionnement.

L'émergence d'un réseau d'approvisionnement d'origine informelle a eu pour conséquence de valoriser les espaces agricoles le long des faubourgs fréquentés. Ainsi avant les années 1970, se dessine dans un rayon de $80 \mathrm{~km}$ autour de Bamako une zone de production de plus en plus variée à destination de la ville, centrée sur les axes d'entrée de Bamako. En 1978, l'émergence d'une seconde génération de véhicules appelés Sotrama ${ }^{15}$ marque la densification du trafic vers les nœuds commerciaux qui ont émergé. En effet, la production villageoise vers la ville s'intensifie et la demande se fait plus forte. Les villages cités précédemment ont un avantage sérieux en terme d'accessibilité puisque depuis 1978, ils sont desservis environ toutes les heures par les transports collectifs du secteur artisanal ${ }^{16}$.

41 Les transformations spatiales de la ville ${ }^{17}$ ont modifié le volume et la répartition des modes de déplacements, tandis que le réseau de transport en commun a contribué à l'accessibilité de nombreux points du territoire. Les distances parcourues s'allongent et les moyens de transports mieux adaptés permettent de se déplacer plus vite et plus loin. En tant que lieu d'occupation ancien et précurseur des productions agricoles vers la ville, les villages de concessions rurales et les sites à vocation maraîchère cités plus haut voient leur rôle se renforcer. Ils constituent des lieux d'innovation et participent à 
la diffusion de nombreuses aires commerciales dans la zone. Établis sur un réseau déjà bien rodé, ces villages bénéficient le plus souvent de l'effet " goudron » et instaurent une hiérarchie en diversifiant leurs fonctions et la gamme de produits délivrés.

L'ampleur du trafic, la croissance de la demande urbaine en vivrier marchand et en bois énergie confirme, dans les années 1980, la constitution d'un véritable réseau d'approvisionnement. Les circuits réguliers de transporteurs et leur intensification depuis 1978, ont engendré une augmentation de la production, surtout maraîchère à l'échelle villageoise. D'autre part, la proximité de forêts classées comme celle de La Faya et la croissance des besoins urbains en énergie domestique ont incité de nombreux paysans à exploiter la forêt et à faire du charbon. De nombreux villages non accessibles et trop éloignés pour espérer commercialiser leurs produits sur les marchés situés sur les routes goudronnées décident de créer leur propre marché. Sur ce modèle ce sont par exemple treize nouveaux marchés qui sont crées entre Kassela et Tiélé de 1975 à 2000. On peut y différencier les foires comme Dialakorobougou et Mountougoula, qui sont proches de la route et qui profitent d'un effet "rupture de charge " et d'un passage important de clientèle vers Bamako; et des marchés « de l'intérieur » comme N'Gouraba et Mounzoun qui, grâce a une production importante de bois et charbon sont à l'origine de flux réguliers de commerçants et de l'implantation de circuits de transports durables sur des pistes pourtant rurales et pas toujours accessibles.

La densification des foires dans l'espace périphérique et le développement de fonctions de ravitaillement de la ville ont eu pour conséquence l'intensification des échanges et des rapports de Bamako avec son arrière pays immédiat. Dès lors, l'éloignement des axes routiers n'est plus discriminant et permet le développement de petits sites d'exploitation villageoise dans un rayon de $100 \mathrm{~km}$ autour de Bamako. Les foires constituent à ce stade la face visible de l'émergence d'une ceinture vivrière et productrice de bois énergie.

\section{Densification et extension du réseau de foires}

44 Les fonctions des villes ou villages accueillant une foire se complexifient. Pour les producteurs, la foire est toujours un débouché fiable, cependant ils y effectuent également leurs achats personnels, dès les transactions achevées. Pour les commerçants urbains, certes les foires ont la capacité d'offrir une large gamme de produits agricoles et en quantité, cependant les trajets de Bamako vers sa périphérie sont aussi l'opportunité de ravitailler les magasins villageois en produits manufacturés. Les trajets allers et retours sont ainsi rentabilisés au maximum et les liens ruraux/ urbains deviennent multifonctionnels. Dans les capitales régionales, les motifs de déplacement les jours de foire sont de plus en plus variés : administratifs, familiaux, débouchés agricoles, accès à une gamme de biens ou de services. Ainsi à Kati, c'est désormais une trentaine de villages dans un rayon de $30 \mathrm{~km}$ qui approvisionnent les deux marchés hebdomadaires. Loin d'avoir perdu son rôle faute de terres cultivables, Kati concentre désormais la production et attire les commerçants, acteurs de l'approvisionnement de Kati et de Bamako. La demande urbaine s'accroissant, de nombreux villages autrefois peu impliqués dans le marché urbain, s'investissent dans le secteur, jusqu'à créer leur propre foire. Parmi la trentaine de villages qui ravitaillent les deux marchés de Kati, 6 villages accueillent désormais une foire, augmentant ainsi les débouchés pour les producteurs spécialisés dans le maraîchage. Les producteurs rencontrés sur les marchés de Kati se rendent sur 2 ou 3 autres foires les autres jours de 
la semaine. La densification du réseau de foires fait partie du phénomène le plus visible dès les années 1980.

L'émergence de nouvelles foires, au lieu d'affaiblir les foires anciennement établies a pour effet de les renforcer dans leur rôle. Dans chacune de nos enquêtes sur le site de Kati ou des villages producteurs, le choix d'une nouvelle aire de commercialisation par un producteur est toujours le fruit d'une stratégie de multiplication des débouchés, plutôt qu'une volonté d'évitement ou de contournement d'une foire longtemps fréquentée. En effet, les liens de fidélisation noués avec les commerçants participent à la sécurisation de l'activité, ou du moins à sa perception. L'opportunité de meilleurs revenus, si elle se confirmait, ne suffirait pas à remettre en cause ces partenariats dans un temps court. D'autre part, la capacité d'attraction des foires anciennes comme Kati est fondée sur sa fonction de débouché commercial mais aussi sur sa capacité à délivrer une gamme de biens et services diversifiée. Tout comme Kati, les capitales régionales qui accueillaient une foire-exposition ${ }^{18}$ pendant la période coloniale n'ont pas perdu ce rôle.

Figure 6 - Foire de Kati

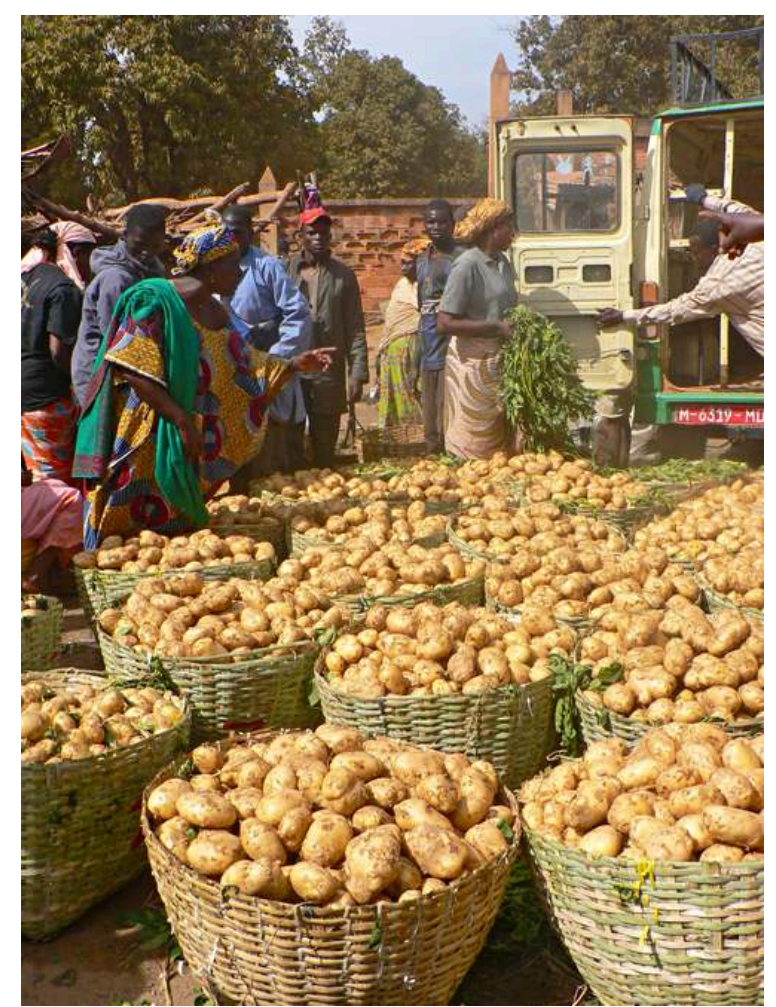

Une commerçante rassemble ses achats pour les transporter à Bamako

Source : G. Raton, juin 2007

Dans le périmètre irrigué de Baguinéda, la riziculture reste la culture principale, mais depuis l'Indépendance des actions valorisant le maraîchage ont fait de ce périmètre un centre important de fourniture de tomates, oignons, gombo, choux, aubergines et mangues vers la ville. Baguinéda, qui est le village le plus accessible à partir de la route goudronnée, est devenu un lieu central d'approvisionnement en produits maraîchers. A tel point que la fréquence hebdomadaire usuelle n'a plus cours. C'est désormais tous les jours que les commerçants ou encore les femmes de producteurs acheminent les 
marchandises jusqu'à Bamako. Au niveau administratif, Baguinéda accueille les habitations des fonctionnaires et les bureaux de l'office des Périmètres Irrigués de Baguinéda (OPIB). Le lieu dit "Baguinéda Camp», qui se situe sur l'axe menant directement à la route goudronnée, a développé des fonctions de marché et de redistribution. On peut observer la présence d'un poste de régulation des transports en commun, d'un hall de marché quotidien, et de lieux de centralisation des productions en gros avant diffusion vers Bamako. Les avantages comparatifs du secteur de rente et de l'approvisionnement vivrier, la forte croissance démographique et la proximité de Bamako ont contribué à faire de Baguinéda un village producteur important pour l'approvisionnement régulier des marchés de la ville. Ils constituent désormais, selon l'OPIB, le principal débouché des productions maraîchères. Bamako est en effet un débouché sûr : la ville est située près des lieux de productions et la structure étatique de gestion du périmètre constitue une garantie. L'amélioration agricole, les techniques d'encadrement font de ce périmètre, malgré des débuts difficiles, un lieu d'innovation agricole et de transmission des techniques. L'influence de la ville se manifeste par la présence de personnel administratif et technique sur le périmètre irrigué de l'OPIB. On recense désormais 22 villages riverains, tous insérés dans les circuits urbains. L'OPIB comptait en 1998 environ 22000 habitants sur l'ensemble du périmètre.

Aujourd'hui, le réseau de foires s'étend dans un rayon de $150 \mathrm{~km}$ autour de Bamako. Les cinq axes goudronnés qui desservent Bamako en formant un réseau routier en étoile, permettent de relier la ville aux foires. Les foires les plus accessibles sont celles situées sur ces axes goudronnés. Sur chacun, on dénombre de trois à huit foires. La distance maximale à Bamako est de $150 \mathrm{~km}$ (Banamba, Konobougou, Kolokani, Siboba, Kouré Malé). Leur répartition est régulière. Depuis les années 1980, on note une stagnation du nombre d'aires commerciales sur ces axes principaux. Seuls quelques points de vente se sont implantés, profitant des flux de passagers pour délivrer des marchandises. Leur mode de fonctionnement est celui du commerce de détail et n'est pas, pour cette raison, assimilable aux systèmes de foires. Cependant, on peut observer une tendance à l'extension du périmètre d'approvisionnement sous une autre forme. Il s'agit de foires de capitales régionales (Ségou, Sikasso, Niono, Didiéni) qui attirent saisonnièrement des commerçants de Bamako. Cette tendance s'affirme mais est à différencier également du réseau de foires. En effet, d'une part les commerçants qui s'y rendent ne sont pas des habitués des foires de la périphérie et concentrent leurs activités sur le commerce transfrontalier. D'autre part, le mode de transport privilégie la différenciation entre transport de passagers et transport de marchandises, assurés respectivement par cars (sociétés nationales) et par gros-porteurs. L'intérêt pour ces espaces d'approvisionnement est à surveiller et pourrait constituer à l'avenir un mode de ravitaillement attractif. La mutation des modes de transport participerait dans ce cas à l'allongement des distances d'approvisionnement ainsi qu'à l'extension de la forme du réseau.

Les autres foires, localisées sur les pistes rurales, sont plus nombreuses. Leur accès est possible à partir des axes principaux. Chaque axe dessert de une à quatre pistes rurales où sont localisées, comme organisées le long d'un couloir, jusqu'à 13 foires. Distantes de $10 \mathrm{~km}$, parfois moins, elles se succèdent sans pourtant se concurrencer directement puisque la fréquence de marché est hebdomadaire. Cette concentration peut être étonnante, pourtant elle répond à une logique de circuits de transports. Chaque piste rurale correspondant à une ligne avec plusieurs terminus. C'est sur ces pistes que la 
densification du réseau est la plus visible. Lorsque qu'un de ses sous axes est particulièrement dynamique en terme de trafic, les créations de nouvelles foires sont fréquentes. Le réseau se densifie (lorsqu'une foire est créée entre deux foires préexistantes) et s'allonge (lorsque que, pour la desservir, le terminus de la ligne est prolongé).

Figure 7 - Tableau récapitulatif des foires par axes routiers majeurs et « sous-axes »

\begin{tabular}{|c|c|c|c|}
\hline $\begin{array}{ll}\text { Axes } & \text { routiers } \\
\text { majeurs } & \end{array}$ & Localisation & $\begin{array}{l}\text { Nombre de } \\
\text { sous-axes } \\
\text { desservis }\end{array}$ & $\begin{array}{l}\text { Sous-axes menant aux foires } \\
\text { hebdomadaires situées sur les } \\
\text { pistes rurales }\end{array}$ \\
\hline $\begin{array}{l}\text { Bamako-Fana- } \\
\text { Segou }\end{array}$ & $\begin{array}{l}\text { A l'est de Bamako, vers } \\
\text { Mopti }\end{array}$ & 4 & $\begin{array}{l}\text { Baguinéda } \\
\text { Tyélé } \\
\text { Kassela - Bassian } \\
\text { Fana - Dioila }\end{array}$ \\
\hline $\begin{array}{l}\text { Bamako-Koulikoro- } \\
\text { Banemba }\end{array}$ & $\begin{array}{l}\text { Au nord est de Bamako, vers } \\
\text { la frontière mauritanienne }\end{array}$ & 3 & $\begin{array}{l}\text { Moribabougou - Kola } \\
\text { Kourikolo - Kouba } \\
\text { Boron - Touba }\end{array}$ \\
\hline $\begin{array}{l}\text { Bamako-Kati- } \\
\text { Kolokani }\end{array}$ & $\begin{array}{l}\text { Au nord et au nord ouest de } \\
\text { Bamako, vers la frontière } \\
\text { mauritanienne et Kayes }\end{array}$ & 5 & $\begin{array}{l}\text { Kati - Faladié } \\
\text { Dio - Kassaro } \\
\text { Kati - N'Galamadibi } \\
\text { Safo - Donoumania } \\
\text { Safo - Donébougou }\end{array}$ \\
\hline $\begin{array}{l}\text { Bamako- } \\
\text { Oulessebougou- } \\
\text { Sidoba }\end{array}$ & $\begin{array}{l}\text { Au sud de Bamako, vers } \\
\text { Sikosso }\end{array}$ & 7 & $\begin{array}{l}\text { Simidji - Dangassa - Gouala } \\
\text { Dialakoroba - Freintoumou - } \\
\text { Baga } \\
\text { Hérémakono - Falan } \\
\text { Safébougoula - Kangolé } \\
\text { Ouelessebougou - Sanankoro - } \\
\text { Djitoumou } \\
\text { Sidoba - Dogo } \\
\text { Selingué }\end{array}$ \\
\hline $\begin{array}{l}\text { Bamako-Siby- } \\
\text { Narena }\end{array}$ & $\begin{array}{l}\text { Au sud est de Bamako, vers } \\
\text { la frontière guinéenne }\end{array}$ & 3 & $\begin{array}{l}\text { Bancoumana - Kangaba } \\
\text { Bancoumana - Karan } \\
\text { Nafadji - Kalakana }\end{array}$ \\
\hline
\end{tabular}

Source : G. Raton, enquêtes personnelles

Outre la distinction de ces "couloirs de passage ", à l'échelle du réseau de foires, l'intégration de nouveaux villages ou d'unités agricoles dans le circuit urbain n'est plus spatialement continue. La multiplication des foires se poursuit sur les axes déjà valorisés, créant des différenciations majeures, entre sous axes intégrés dans le marché 
urbain et marges bénéficiant des échanges sans en faire partie intégrante. Ces différenciations peuvent se lire dans le paysage. Le parcellement en concession rurale a engendré des paysages aux formes géométriques et un cloisonnement d'espaces de culture jusque là inconnu. On observe au sein des terroirs villageois de plus en plus d'habitats avec un toit de tôle. Les vagues de migration vers la ville ont créé des rapports étroits entre nouveaux citadins et villages d'origine. Les déplacements entre familles citadines et villageoises sont encouragés par le développement des lignes de transport régulières ${ }^{19}$ et peuvent s'accompagner d'investissements immobiliers pour la retraite ou comme maison secondaire. Certains fonctionnaires font quotidiennement le trajet de la périphérie vers Bamako. Les villages situés entre plusieurs entités administratives majeures comme Koulikoro, Kati et le District deviennent des lieux de résidences de notables. L'augmentation des flux de la ville avec sa périphérie immédiate, que ce soit via des migrations définitives, temporaires ou journalières, a fait émerger un nouveau lien entre ruraux et urbains, jusque là circonscrit aux impressions de main mise des citadins sur les terres agricoles.

\section{Les foires marquent le paysage et la composition urbaine du territoire}

Actuellement, les conflits entre recherche de surfaces constructibles et recherche de terre pour l'exploitation agricole se font plus pressants. L'affirmation de fonctions de production puis de commercialisation est remise en cause en de nombreux points de l'espace périphérique. Cette partie est l'occasion de voir quels sont les processus qui remettent en cause la présence d'une foire. Nous verrons, selon un gradient ville/ périphérie, les modalités de changement de ses fonctions.

\section{L'urbanisation croissante remet en cause le fonctionnement des foires}

51 La densification du réseau de foires hebdomadaires, phénomène observé et décrit depuis les années 1980, a des limites. En effet, on constate que les foires les plus proches de la ville sont progressivement désertées par les commerçants. Ce phénomène peut être attribué à l'augmentation de la pression sur les ressources naturelles et à la diminution des terres à usage agricole, sous l'effet de la croissance urbaine (passage de concessions rurales à des terres d'habitation). Aujourd'hui, la loi interdit la concession de terres agricoles dans le périmètre urbain. Dès lors, lorsque les concessions rurales sont affectées en lieux d'habitation, aucun retour à la terre n'est possible, les seules vocations admissibles sont administratives, commerciales, industrielles ou à usage d'habitation. La conséquence est une diminution des productions commercialisables et, par ricochet, une baisse de la fréquentation des commerçants. Les foires implantées disparaissent alors ou retournent à des fonctions locales, d'échange de productions villageoises.

En l'espace de 40 ans (1945 à 1985), des villages créés pour leurs fonctions agricoles sont rattrapés par l'urbanisation. C'est le cas de parcelles de maraîchage en milieu urbain, qui subsistent mais ont de plus en plus vocation à occuper les interstices urbains. C'est également le cas des fermes d'Etat, implantées en périphérie du District, dont la superficie à tendance à diminuer. Par exemple, la ferme de Sotuba s'étendait sur 1000 
hectares à sa création, aujourd'hui on ne compte plus que 268 hectares, le reste ayant été attribué comme terre à usage d'habitation par la mairie ${ }^{20}$. On retrouve la même tendance pour les espaces situés sur les rives du Niger, exploitées pour la culture maraîchère et qui sont désormais contigus aux quartiers urbains. A proximité de Koulikoro par exemple, le village de Moribabougou a été loti à la fin des années 1990. Avant son lotissement, ce village possédait un marché hebdomadaire. Les habitants étaient massivement investis dans le maraîchage et écoulaient leur production sur la foire. Sur cet axe, l'étalement urbain fut important de 1987 à 1998. Le village de Titibougou puis celui de Moribabougou, jointifs, ont été lotis. En 10 ans (de 1987 à 1998), la population a été multipliée par deux. Moribabougou comptait 4500 habitants en 1998. On peut attribuer cette forte croissance démographique au développement de la fonction résidentielle, ainsi qu'à la création d'un poste de contrôle du trafic (gendarmerie, douane, contrôle forestier) qui a eu tendance à fixer la population. Le lotissement de parcelles agricoles et la présence d'une élite urbaine travaillant à Bamako ou Koulikoro a fortement modifié le fonctionnement de la foire. D'une part, les quantités commerciales sur la foire ont considérablement chuté. Seuls les villages périphériques continuaient à assurer le ravitaillement. D'autre part, les besoins de la population ont radicalement modifié le fonctionnement temporel du marché. La clientèle professionnelle a disparu au profit d'une clientèle de quartier. En l'espace de deux ans, la foire s'est transformée en marché de quartier, fonctionnel tous les jours, principalement aux heures de sorties des offices. Ce phénomène de transformation de foire en marché de quartier est courant dans la périphérie proche de Bamako. Le travail de terrain nous a permis de retracer ces fonctions anciennes qui ne sont plus visibles actuellement dans le paysage. Seul le lien qui existe encore entre commerçants de quartiers et producteurs de villages périphériques permet de confirmer la présence ancienne d'une foire.

Compte tenu de la croissance démographique (fig. 1), il est indéniable que la densification du bâti et l'étalement urbain se trouvent être en conflit avec les fonctions agricoles. "Pour celles (les villes) qui ont une existence ancienne, la proximité de ressources agricoles a été, pendant des siècles, indispensable au maintien d'une population concentrée, étant donné la précarité et la lenteur des moyens de transport. Dans ces conditions, tout développement urbain entre directement en concurrence avec l'utilisation agricole du sol.» (Bastie, 1980, p. 57). Ce qui est intéressant ici, c'est que plutôt que de péricliter, la foire laisse place à une autre forme de marché plus centré sur des fonctions locales d'approvisionnement. On peut ainsi affirmer que la fonction commerciale ancrée dans les pratiques n'a été à aucun moment dissociée du lieu. Elle marque durablement les usages de l'espace.

\section{Des foires en perte de vitesse mais des unités agricoles qui intensifient leur lien à la ville}

La vie d'une foire n'est pas linéaire. La capacité d'attraction est variable et peut mettre en péril la viabilité économique de l'aire commerciale. En effet, la multiplication des foires a permis aux commerçants urbains de diversifier les lieux d'approvisionnement. Ces marchés offrent de nombreux débouchés pour les producteurs mais créent également un climat de concurrence accru: les exigences des commerçants se renforcent (fig. 6). Afin d'assurer un fonctionnement régulier de leur activité, ils choisissent les marchés capables de fournir une large gamme de produits, en quantité 
suffisante, et de bonne qualité. Le dynamisme d'une foire, et donc sa pérennité, dépend de sa capacité à attirer un ensemble d'acteurs de la chaîne d'approvisionnement ${ }^{21}$.

Parmi les foires étudiées, nous avons recensé un ensemble de foires soumises à une grande variabilité de flux. Ces foires sont respectivement des aires de vente nouvellement créées par décision communale et les foires dont la pérennité économique est fortement menacée par la proximité d'une foire dynamique. Ces foires en perte de vitesse sont frappées par l'absence de flux de commerçants urbains. Les producteurs sont donc confrontés à des problèmes de débouchés. Nous avons recensé 20 foires dans ce cas (Fig. 5).

Comme nous l'avons vu précédemment, la distance à la ville est un facteur important. L'étalement urbain peut remettre en cause la fonction commerciale et modifier l'organisation classique. Ainsi, les foires situées sur l'axe Baala, le long du fleuve Niger au Sud de l'agglomération, sont toutes confrontées à une diminution des flux. Sur cet axe, on retrouve les villages de Madina ${ }^{22}$, Banco Coura, Siene, Tourela, Kabé et Baala. Pourtant cet axe fut très fréquenté dans les années 1980. Situés le long du fleuve, ces villages ont développé de nombreux jardins maraîchers et ont participé à l'approvisionnement des marchés de la capitale. Les flux de transporteurs étaient réguliers il y a encore une dizaine d'années. Depuis, et en l'espace de très peu de temps, les transporteurs ont cessé de fréquenter cet axe, les marchés hebdomadaires ont perdu de leur attractivité. Désormais, ce sont les flux de charrettes et de pousse-pousse vers la ville qui sont la marque visible d'une intégration au circuit urbain. Pourtant, dans ces villages, rien n'indique une urbanisation croissante : la piste, en mauvais état n'est pas goudronnée, les villages sont d'allure classique. Seule la densité de petits jardins le long du fleuve indique une activité commerciale intense avec la ville. Après une période de forte production, d'attraction hebdomadaire de transporteurs et de commerçants pour l'achat de produits maraîchers, ces villages n'ont pas rompu le lien à la ville. Désormais, ce sont eux qui assurent la production et le transport de marchandises vers les marchés des quartiers périphériques. Ils sont retournés vers des modes de déplacements non motorisés et, sur le marché, ce sont désormais les échanges locaux qui dominent. Paradoxalement, leur lien à la ville s'est renforcé. Les commerçants et transporteurs ont augmenté leur rayon de déplacements et ce délaissement leur a donné l'opportunité d'assurer eux même la commercialisation. La trentaine de kilomètres qui les séparent de la ville est en effet signe d'augmentation des prix. Peu à peu, les producteurs ont préféré assurer un voyage pénible vers la ville et augmenter leurs marges bénéficiaires.

Cet exemple, unique à l'échelle du réseau par le nombre de foires contiguës " affectées », montre deux choses. D'une part, la proximité à la ville remet en cause le modèle marchand des foires. En deçà d'une certaine distance (30 à $40 \mathrm{~km})$, les transports non motorisés se substituent aux flux de transporteurs et de commerçants. Il existe une distance en dessous de laquelle les producteurs ont tout intérêt à réduire le périmètre d'attraction de la foire pour assurer eux même la livraison et la vente au sein de marchés urbains. D'autre part, cet exemple de décroissance du réseau de foire sur l'axe Baala montre que les évolutions positives ou négatives de la forme du réseau de foires marquent le paysage. L'absence d'un des acteurs de l'approvisionnement modifie la dynamique de villages centrés sur la production et la commercialisation. La dynamique commerciale impulsée ne bénéficie plus au village d'accueil mais peut 
permettre de resserrer les liens coutumiers avec les communautés paysannes voisines, sans pourtant remettre en cause les liens à la ville.

La dynamique des foires est lisible sur la figure 5 où sont localisées les foires qui ne font pas l'objet de flux de commerçants urbains, soit parce qu'ils sont en perte de vitesse, soit parce qu'ils sont récents. Nous n'avons pas différencié ici les deux, considérant le déplacement des commerçants urbains vers les foires comme un élément de définition de la foire. L'essentiel étant de les localiser comme des éléments potentiels d'accroissement ou de réduction du réseau.

\section{Evolution des foires vers des fonctions commerciales indépendantes du ravitaillement urbain}

Au delà de l'intégration d'unités agricoles au circuit urbain, la présence d'une foire à l'échelle villageoise est le signe de la croissance de fonctions commerciales. Ceci pose la question de l'adéquation de ces deux fonctions. En effet, les flux engendrés par la foire encouragent la fixation d'une fonction commerciale propre au village d'accueil, pas toujours compatible à la fonction agricole sur le long terme.

Des exemples nous montrent que la fonction commerciale prend parfois le pas sur la fonction agricole, sans que la structure même de foire ne soit remise en cause. A Kassela par exemple, les concessions rurales concédées en 1960 sont aujourd'hui devenues des zones d'habitations. Ceci marque l'accroissement de la fonction résidentielle dans ce village et pourquoi pas le début d'une inflexion de son rôle de ravitaillement de la ville. Pourtant, on remarque une intensification des échanges. En effet, comme à Baguinéda et Siby, l'intensification des flux dès 1978 a changé les fréquences de ravitaillement: le jour de foire n'est plus le seul moment de commercialisation, bien qu'on y observe toujours un pic hebdomadaire de fréquentation. L'implantation urbaine pour usage d'habitation n'a donc pas altéré les capacités de production. Il faut rappeler l'originalité de ces espaces. Tout d'abord, ils sont situés à 30 et $45 \mathrm{~km}$ de Bamako. Bien que l'on observe le développement de la fonction résidentielle, l'étalement urbain n'est pas encore une préoccupation majeure. D'autre part, ils sont l'objet depuis l'époque coloniale d'un encadrement technique important : par les services forestiers pour le village de Kassela, par l'administration de l'OPIB pour Baguinéda, et par les responsables des plantations de Samanko pour Siby. Malgré quelques tentatives, ils n'ont pas subi de vente massive du parcellaire comme nous l'avons constaté en marge de la ville. L'ancienneté de l'occupation et de l'exploitation des terres a sans doute permis de fixer davantage les activités de production. Enfin, les pratiques de commercialisation journalières ont renforcé le rôle de ces villages comme pôles commerciaux ou de distribution. La fonction de production n'est plus la seule à l'œuvre, la fonction commerciale semble s'imposer. En effet, l'augmentation de la population (fig. 8) et la présence d'une communauté de notables travaillant en ville ont eu tendance à modifier progressivement les fonctions du marché. On remarque, dans ces villages situés le long des axes routiers fréquentés, l'émergence de marchés quotidiens en plus de la foire hebdomadaire. Ces marchés ont pour vocation le ravitaillement quotidien des ménages vivant dans le village. A cette structure commerciale s'ajoutent le plus souvent des magasins fixes, jouant le rôle à la fois de stockage des productions destinées à la ville et vendues le jour de foire, et de « magasins de quartier » capable de ravitailler les habitants vivant sur place, de plus en 
plus investis dans les activités commerciales. Ces infrastructures sont adaptées à une demande dite rurale de plus en plus importante. Si ces villages n'ont pas atteint le seuil symbolique des 5000 habitants $^{23}$, ils ont de plus en plus des allures de quartiers, bien qu'isolés. Leurs fonctions commerciales leur ont conféré des fonctions de point de rupture de charge entre approvisionnement urbain et approvisionnement rural.

Figure 8 - Croissance démographique des villages de Bauinéda Camp, Kassela et Siby (1976-1998)

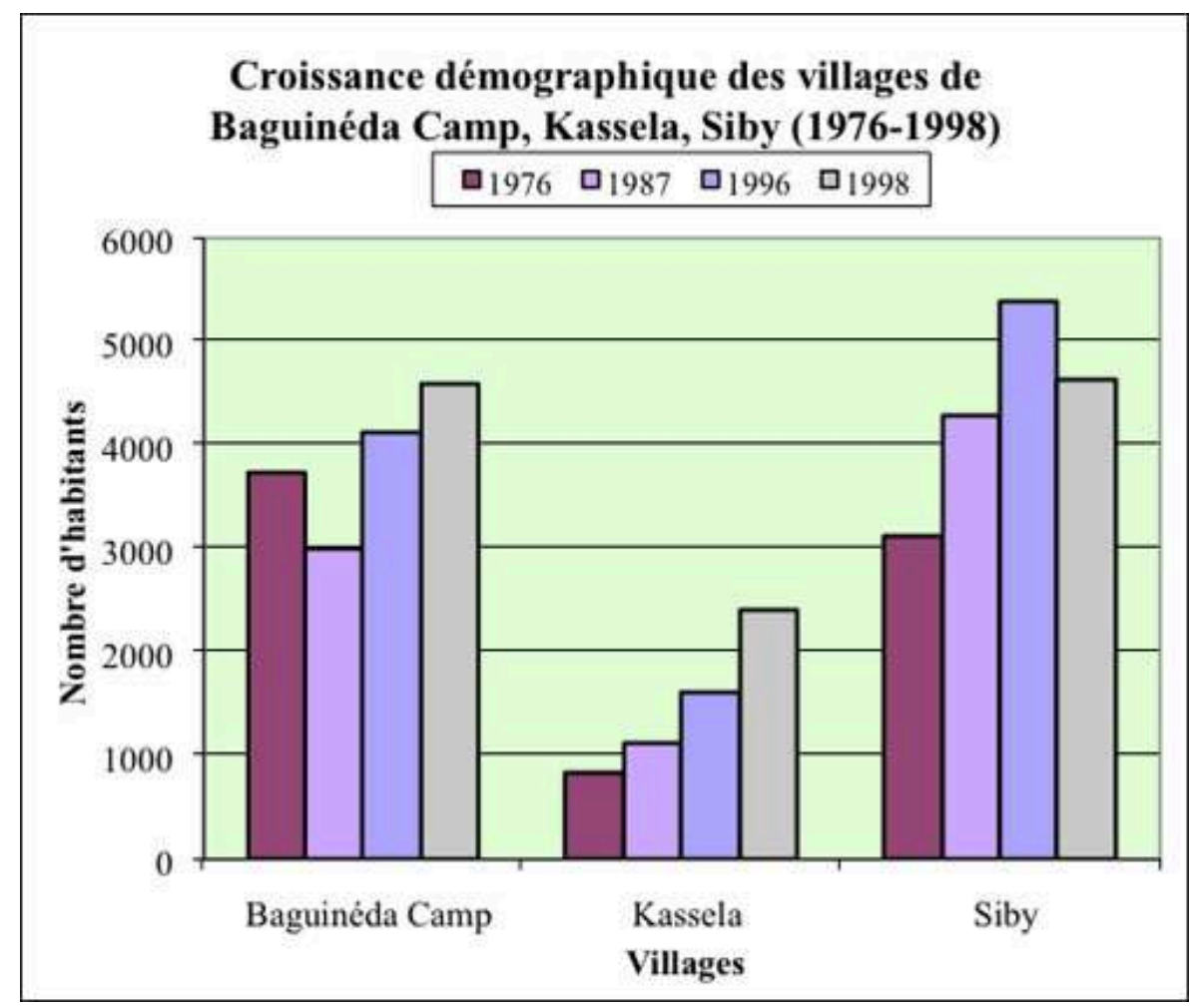

Source : Données DNSI Mali

Suivant le gradient à la ville, le processus d'intégration d'unités agricoles au marché urbain varie fortement. La foire a un rôle d'intégration de nouveaux acteurs de la communauté paysanne dans l'approvisionnement de la ville. Ce rôle peut être complété par une fonction d'approvisionnement des villageois fréquentant la foire. Face à la pression démographique et à l'extension urbaine, la fonction de ravitaillement s'exerce au niveau de marchés de quartiers ou directement sur les marchés de la capitale. Enfin, une fois les espaces intégrés au milieu urbain, la fonction commerciale est la seule à subsister.

\section{La foire : un marqueur de la composition urbaine?}

Le réseau de foires a deux limites distinctes: d'une part une limite qu'on pourrait appeler "extérieure», d'autre part une limite avec l'espace urbanisé. La limite extérieure est définie par la distance au-delà de laquelle soit le modèle marchand de la foire n'a plus cours, soit l'intégration de nouvelles unités agricoles n'a pas eu lieu, ou encore lorsque l'approvisionnement vise un autre pôle urbain. Ces deux limites sont mouvantes. En effet, comme nous l'avons vu, l'intégration de nouveaux villages au système d'approvisionnement est un processus en cours, même s'il concerne davantage 
les espaces au sein des pistes rurales que les axes routiers majeurs. De même, dans la frange urbaine, les espaces de cultures sont réduits au bénéfice du bâti. Les foires les plus proches de la ville sont amenées à changer de forme. L'étalement spatial de la ville de Bamako gagne actuellement du terrain sur les espaces agricoles de la frange urbaine. La croissance démographique est continue et les besoins plus grands; on peut émettre l'hypothèse que le bassin d'approvisionnement de Bamako via les foires est amené à croitre au rythme de la croissance urbaine. Le périmètre d'extension d'une foire, tout comme celui d'un bassin d'approvisionnement classique, est donc un facteur de compréhension des dynamiques urbaines.

63 De même, la transformation de foires en marchés de quartiers montre que la fonction commerciale subsiste malgré une diminution des flux d'approvisionnement issus d'un hinterland en phase d'urbanisation. L'évolution des limites du réseau de foires dans les franges urbaines montre également les dynamiques urbaines en cours. Les foires marquent donc durablement le paysage notamment dans ses limites avec l'espace urbain. La production de formes commerciales qui, ont l'a vu, ont tendance à subsister, en fait un marqueur des formes urbaines.

\section{Conclusion}

Les grandes étapes de l'histoire urbaine de Bamako ont durablement marqué le paysage périphérique. Les premières actions de mises en valeur ont influencé la diffusion et l'institutionnalisation d'un mode de commerce: la foire. Sa diffusion est le fruit de politiques de mise en valeur agricole issues de la période coloniale et de l'accès à l'Indépendance. Elles visaient à faire émerger d'une part une communauté paysanne active pour la fourniture de produits pour la ville et, d'autre part, à intégrer l'élite urbaine à l'organisation de cet approvisionnement. Cette mise en valeur initiale de la périphérie a fortement marqué les terroirs ruraux et leur intégration au circuit urbain. L'absence de rupture majeure entre politique coloniale et politique de l'Indépendance en matière de fixation de la population agricole et de sécurisation du réseau d'approvisionnement explique sans doute que les formes d'intégration d'unités villageoises au réseau d'approvisionnement soient lisibles encore aujourd'hui dans l'espace.

L'implantation de fermes citadines dispersées marque une étape puisqu'elle s'assimile à une consommation de l'espace agricole, ce qui complexifie sa gestion. Pourtant, situées au contact direct des communautés paysannes, elles ont joué un rôle de diffusion de l'agriculture «moderne » dans les usages et les mentalités. Les communautés villageoises ont élargi leurs perspectives économiques en associant aux cultures d'autoconsommation la possibilité de produire pour la ville, en étant soit soutenues par des opérations de développement rural, soit sur des initiatives individuelles. Les petits jardins maraîchers, à la manière de ceux implantés en ville pendant la colonisation, sont devenus en périphérie des sources de revenus réguliers pour les paysans et sont le signe d'une intensification de l'exploitation sur de petites parcelles. De plus, l'intégration au marché urbain et la diversification des besoins ont tendance à augmenter le temps consacré à l'activité. Les productions sont plus importantes et la fréquentation de plusieurs lieux de vente se démocratise.

Ces éléments historiques, nous ont permis de comprendre les formes actuelles du réseau de foires. Original et évolutif, ce réseau est le reflet de la performance des 
systèmes de transport desservant les territoires, des potentialités agricoles, de l'existence du lien à la ville. L'efficacité des systèmes de foires induit un enjeu en termes de pérennité des débouchés des productions agricoles et de revenus villageois. La rentabilité de l'activité est dépendante du lien étroit tissé avec les commerçants urbains. La présence de ces aires commerciales impulse une dynamique villageoise qui dépasse désormais une simple logique d'écoulement des productions à but commercial. Les activités de services se développent au sein des villages accueillant une foire. Les points de vente se densifient et se diversifient, favorisant une circulation accrue des marchandises notamment de type manufacturé vers les foires, puis vers un réseau de boutiques secondaires villageoises. Stimulés par la présence d'une aire économique attractive, de nouveaux acteurs villageois se lancent dans le commerce, propice à la création de liens entre foires et entre villages, jusque là inexistants.

L'analyse de ces aires de vente révèle plusieurs processus. Tout d'abord, elle met en lumière l'ampleur de l'intégration de villages ou d'unités familiales au circuit urbain. En effet, chaque foire mobilise un hinterland composé d'une quinzaine de villages, soit autant d'acteurs qui contribuent à la sécurisation des besoins urbains. En retour, ces acteurs bénéficient d'un réseau d'accès à des produits manufacturés. D'autre part, les flux émis et attirés par une foire font émerger en périphérie de Bamako des "pôles commerciaux » faisant office de places centrales, dont le poids s'affirme au cours des années 1980. Ils structurent la périphérie et c'est autour de ces centres que se densifie le réseau. Enfin, le réseau de foires est évolutif. Sa forme est fortement dépendante des processus d'urbanisation. Ils limitent son extension en frange urbaine et impulsent une logique d'extension sur de nouveaux espaces ruraux, à intégrer dans les circuits d'approvisionnement. A l'échelle d'une foire, nous avons mis en évidence la mutation de la forme des foires: en termes de temporalité (quotidien, hebdomadaire), de fonctions (production, commerce), de direction des flux et de modalités de transport. Ces changements en cours permettent d'une part d'identifier les nouveaux mécanismes d'intégration d'espaces (hinterland des foires). D'autre part, ils permettent de mesurer la croissance urbaine. La naissance, la transformation ou la perte de vitesse d'une foire est le signe d'une inflexion des flux d'attraction et dénote le changement de fonction de l'espace en question. En ce sens, l'évolution du réseau de foire est un indicateur de l'urbanisation.

\section{BIBLIOGRAPHIE}

Bastié J., Dezert B., 1980. L'espace urbain. Masson, Paris, 382 p.

Bleneau D., La Cognata G., 1972. Evolution de la population de Bamako. Etudes Maliennes, $\mathrm{n}^{\circ} 3$, p. 26-46.

Chaléard J.-L., Dubresson A., 1999. Villes et campagnes dans les pays du sud, Géographie des relations. Paris, Karthala, 260 p.

Chaléard J.-L., 2002. Marchés et commerce des produits vivriers. Grafigeo, 2001, 91 p. 
Diarra B., Ballo M., Champaud J., 2003. Structure urbaine et dynamique spatiale à Bamako. Edition Donniya, Bamako, $164 \mathrm{p}$.

Djire M., 2006. Immatriculation et appropriation foncière dans une zone peri-urbaine du Mali les avatars d'une procédure (nécessaire ?). Colloque International « Les frontières de la question foncière ", Montpellier, $26 \mathrm{p}$.

Doumbia S., 1982. L'espace périurbain en jeu : l'extension des cultures maraîchères et fruitières à Bamako. Mali, thèse de doctorat de 3ème cycle, EHESS, laboratoire de sociologie et de géographie Africaine, Paris, 385 p.

Gary-Tounkara D., 2008. Migrants soudanais/maliens et conscience ivoirienne - les étrangers en Côte d'Ivoire (1903-1980). L'Harmattan, 343 p.

Lecaillon J., Morrisson C., 1986. Politiques économiques et performances agricoles. Le cas du Mali 1960-1983. OCDE, Paris, 187p.

Piche V., Ouedraogo D., 2000. L'insertion urbaine à Bamako. Karthala, Paris, 206 p.

Rossi-Villien, 1963. Bamako, capitale du Mali. Cahiers d'Outre Mer, nº 64, XVI.

Van Beusekom M., 1997. Colonisation Indigene: French Rural Development Ideology at the Office du Niger, 1920-1940. The International Journal of African Historical Studies, vol. 30, Nº 2 (1997), p. 299-323.

\section{NOTES}

1. Seuls $2800 \mathrm{~km}$ de routes sont goudronnés sur un total de $18000 \mathrm{~km}$.

2. Combustible ligneux à usage domestique.

3. Commerce lié aux échanges au sein d'une économie de subsistance.

4. La population étrangère est principalement d'origine européenne. On recense aussi des populations d'origine libanaises ou métisses.

5. Les mets européens sont prisés au détriment des cultures locales traditionnelles.

6. Coûts importants et lenteur des transports.

7. Le chantier a débuté en 1926 par des travaux de creusement d'un canal à partir de Sotuba. Dans la même optique que l'office du Niger, ces parcelles ont été exploitées par les habitants de «villages de colonisation » créés par transport forcé de colons. Ce périmètre est actuellement appelé Office du Périmètre Irrigué de Baguinéda (OPIB). Des villages ont été inclus dans le périmètre hydro-agricole créé par l'administration française, où ils ont pris la forme de lotissements agricoles.

8. Dans les capitales régionales comme Bamako, Kati et Koulikoro.

9. L'actuel IPR de Katibougou, qui forme des techniciens et agronomes aux secteurs du maraîchage, de la pêche et de la forêt est crée à l'époque coloniale. Situé à $3.5 \mathrm{~km}$ en aval de Koulikoro, «Katibougou devint le berceau de l'arboriculture et du maraîchage au Mali » (Doumbia, 1982, p69).

10. Actuellement, ces marchés sont le lieu de commercialisation de vivrier marchand (produits maraîchers, céréales, produits de cueillette) et de bois énergie.

11. Les premiers villages de concessions rurales sont mis en place en 1960.

12. Tout producteur, même s'il commercialise une partie de sa production, affirmera produire un peu de mil/sorgho pour la sécurisation des besoins familiaux.

13. Par exemple, il s'agit de Sogoniko, Faladié et Magnambougou, actuellement tous intégrés au District de Bamako. 
14. Les «bâchés » sont des véhicules de type pick-up très couramment utilisés dans le transport de marchandises ou de personnes.

15. Le terme «Sotrama » désigne un minibus de transport en commun. Son nom provient d'une société de transport d'Etat qui a fait faillite.

16. C'est le cas de Kassela, Baguinéda, Moribabougou, Siby et Dangassa

17. On estime la superficie de Bamako à 8000 ha en 1986, 12800 ha en 1996 (Diarra, 2003).

18. Kati, Koulikoro, Fana, Dioila sont des capitales régionales insérées dans les circuits de fréquentation des commerçants de Bamako.

19. A différencier des lignes de transport spécifiques aux déplacements les jours de foire.

20. Source: Journal l'Essor, quotidien malien, $n^{\circ} 16204$ du 26 mai 2008, «recherche agronomique, des résultats spectaculaires »

21. Commerçants, producteurs, intermédiaires, consommateurs ou transporteurs.

22. Madina est la foire la plus proche de la ville. Elle se situe à proximité de Kabala, le dernier quartier urbanisé de Kalabancoro.

23. Le seuil de 5000 habitants agglomérés est un critère de définition de la population urbaine au Mali

\section{RÉSUMÉS}

En périphérie de Bamako, la multiplication du nombre d'aires commerciales destinées à approvisionner la ville permet d'interroger le processus d'intégration de nouvelles unités agricoles et de villages aux circuits d'approvisionnement urbain. Le constat de l'inégale répartition de ces marchés et de leur rôle de place centrale pousse à réfléchir sur trois aspects. D'une part, sur les politiques qui ont influencé la diffusion et la normalisation d'un mode de commercialisation, celui des marchés hebdomadaires, localement appelés « foires ». D'autre part, sur l'appropriation de ce mode de commerce par les communautés paysannes et les processus de densification et d'extension du réseau ainsi constitué. Enfin, de la durabilité de ce système commercial dont les formes ont tendance à varier en fonction de la proximité à la ville.

The increase in the number of market places on the outskirts of Bamako which supply the city with food allows one to study how villages and new agricultural units are integrated to the urban supply chains. The unequal distribution of market places on the Bamako periphery and the central role they play for local inhabitants leads one to ponder three questions. The first question concerns the policies which influenced the diffusion and normalization of a selling model characterized by weekly markets, locally known as "fairs". The second question bears on the appropriation of this selling model by peasant communities and on the densification and extension of the resulting network. The third question raises the issue of the sustainability of this selling model, which tends to vary depending on proximity to the city.

\section{INDEX}

Keywords : Bamako, fair, Mali, periodic market, urban food supply, urban fringe

Mots-clés : approvisionnement urbain, Bamako, foire, Mali, marché hebdomadaire, marché périodique, périphérie urbaine 
AUTEUR

GWENAËLLE RATON

Gwenaëlle Raton est doctorante en géographie sous la direction de Jean Louis Chaléard à l'UMR

8586 CNRS-PRODIG - Université Paris 1 et ATER à l'université de Lille I.gwenraton@hotmail.com 